\title{
First video game to treat disease gains FDA okay
}

\author{
The prescription-only game is said to improve attention function in children with attention deficit hyperactivity \\ disorder (ADHD).
}

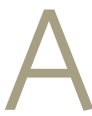

video game that treats disease arrived on the market with a green light issued in June from the US Food and Drug Administration (FDA). The product, developed by Akili Interactive Labs, is the first game-based therapeutic to be approved by the FDA for any condition and the first digital therapeutic approved for ADHD, according the agency.

The move sets a regulatory precedent for other companies in the digital therapeutics space and brings confidence to this fledgling industry. "It shows that the FDA can regulate these interventions," says Jo Masterson, cofounder and CEO of 2Morrow, a company developing digital therapies to help people stop smoking and make other behavioral changes. "We're all super-excited. Any time anyone in the industry validates the ability for digital therapeutics to meaningfully help improve our healthcare, that's good for the whole industry," she says.

Digital therapeutics are software-based devices that treat disease. The treatments may come in the form of information or visual stimuli, for example, and are delivered via a phone, computer or other digital device. Companies that want to claim, or advertise, that their software treats or diagnoses disease typically must receive clearance from the FDA before selling it to consumers.

Getting FDA approval differentiates digital therapeutics from the sea of wellness apps on the market that are available directly to the public. Wellness apps help people track their activities or mood or guide people through healthy activities. Headspace, for example, makes a meditation app that helps people "reduce stress" and "improve mood," according to the company. Since that kind of wellness claim doesn't involve treating disease, Headspace can market directly to the public without FDA review.

Still, some digital therapeutics companies contend that being distinguished as an FDA-approved therapy will pay out in the end. A handful of players in the digital space have already successfully navigated the US regulatory review process. WellDoc in 2014 received FDA clearance for a prescription-only app called BlueStar that functions as a digital assistant for people with type 2 diabetes, helping them determine when to test their blood sugar and how to control it with medication,

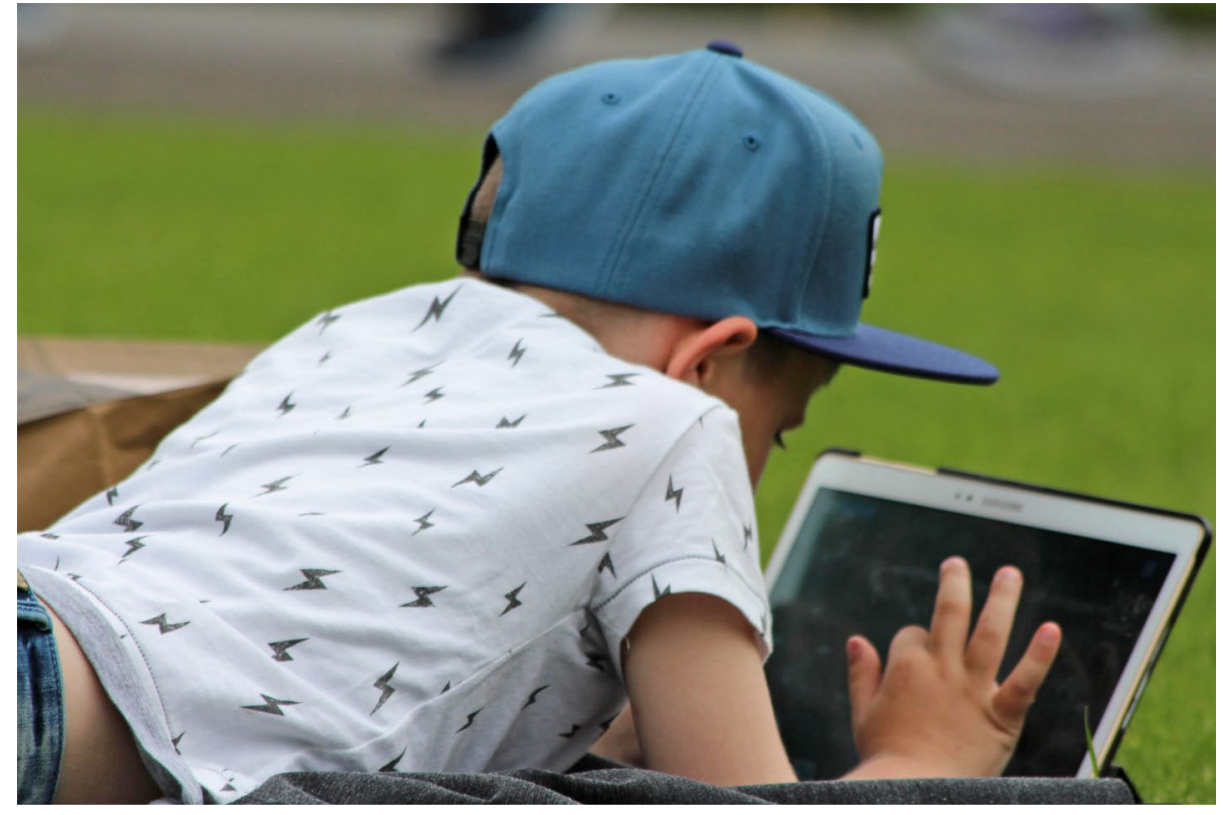

On doctor's orders: playing a video game can deliver treatment for ADHD. Flora Bright / Alamy Stock Photo.

food and exercise. Pear Therapeutics in 2017 received the go-ahead from the FDA to market a prescription-only app that delivers a digital form of cognitive behavioral therapy that helps people with alcohol, marijuana, cocaine and stimulant substance-use disorders stay on top of their cravings. The company has since received clearance to market apps that provide information and counseling to support people with opioid-use disorder and chronic insomnia.

Akili's new software, called EndeavorRx, delivers treatment not as information or counseling, but as an engaging video game. By presenting simultaneous sensory and motor stimuli, the game teaches children with ADHD to manage competing cognitive tasks, shift attention between tasks, and ignore distractors. Akili will price the software roughly within the range of conventional ADHD drugs, says Eddie Martucci, the company's CEO.

Akili had decided from an early stage to make FDA approval part of its business model. "We put the stake in the ground very early, when we founded the company. So in that sense, we're very much like a biotech company," says Martucci. "Our long-term model is that the digital therapeutics industry should have products that are just like medicine in almost every aspect other than interaction. That means the doctor is empowered to prescribe it and insurance companies are empowered to cover it, just like a drug."

Only time will tell if the business model works. "We won't know in a few quarters," says Martucci. It will depend in part on how doctors integrate the game into their care and whether payers will cover it, he says. But Martucci says he is confident that going the regulatory route was worth it. "Nothing has made me waver on this being a good decision," he says. Akili has raised $\$ 120$ million so far.

In making its case to the FDA, Akili presented data from five clinical trials, all of which examined both safety and efficacy. Having clinical efficacy data "turned out to be really important in this novel FDA process," says Martucci. “The vast majority of questions and dialogue [with the FDA] was on the clinical benefit to patients," he says.

Akili claims its product improves cognitive function. The cornerstone of the company's evidence comes from a randomized controlled trial involving 348 children with ADHD, described in February 
in The Lancet Digital Health. Roughly half of the participants in the study played Akili's game while the other half played a sham game created for the purpose of the study. Both groups played 25 minutes per day, 5 days per week for nearly a month.

Trial researchers measured attention improvement based on the children's scores on a computerized attention performance test, called the Test of Variables of Attention (TOVA) Attention Performance Index (API), which was given to the children before and after the intervention period. Children who had been playing EndeavorRx improved their TOVA API test score significantly more than those who played the sham game - an improvement of 0.93 on the test for the experimental group versus an improvement of 0.03 for the control group. Of the children who had been playing EndeavorRx, 36\% no longer showed an attention impairment in at least one measure of attention, compared with $21 \%$ in the control group.

Whether that translates into improved attention function in real life is unclear. "For pretty much anything, you can probably show that doing a certain thing more and more makes you better at that sort of thing, but how well does that transfer over to real-world situations?" says David Cooper, a psychologist and manager of strategic partnerships at Xealth, a digital platform company that integrates digital health tools with electronic health records. "The real question and thing to look at is: Do these kids actually get better at school? Do they focus better and are they better able to regulate their emotions?"

Martucci says he agrees that products need to show benefits outside the treatment, which is why his company's key trial measured efficacy based on improvement on the TOVA API - a long, boring computer-based test - and not improvement on the game itself. A follow-up study that used a parent-reported assessment as an endpoint is in progress.

Additional discussion with the FDA centered on other novel elements of Akili's game and trial designs, Martucci says. For example, the video game adapts to a child's ability and progression in real time. So, unlike a traditional drug, where every pill is the same, Akili's video game therapy is slightly different from one 'dose' to the next and from one patient to another.
Despite the regulatory success of Akili, Pear and others, some decision makers in the digital therapeutics space say seeking the FDA's approval isn't necessary. Makers of smoking cessation apps, for example, are building a body of evidence through clinical trials and finding a market through healthcare providers without the FDA's stamp of approval, says Masterson at 2Morrow. "We know in general that health insurance plans want to pay for FDA-approved interventions - that's a valid point. However, we're getting paid by health plans now through their health and wellness programs, without going that direction," she says. The prospect of approaching the FDA is still on the table for her company, Masterson says, but was not initially part of the business plan.

\section{"The FDA's attention on digital therapeutics during the pandemic suggests that the agency is recognizing them as legitimate, and even necessary, for human health."}

"The issue with the FDA is that the landscape is still a little bit the Wild West," adds Cooper at Xealth. "If you're a company, the biggest thing you want to do is lower the risk of your operation. And right now there's a lot of uncertainty and risk in the digital health landscape. The FDA is very beneficial and helpful, but the process is still new, it changes, and it's still a little bit uncertain," he says.

That uncertainty was enhanced by COVID-19. When the pandemic hit earlier this year, the FDA made a notable change in its digital health policy: as an emergency measure, it would allow digital therapeutics onto the market without regulatory oversight. The change was part of an effort to get mental health care to people during isolation and economic shutdowns, and applies to a wide range of therapies that do not present an undue risk in treating certain psychiatric conditions, including obsessivecompulsive disorder, insomnia, depression, substance-use disorder, autism and ADHD. The policy remains in effect for the duration of the emergency.

Akili was awaiting the FDA's decision on EndeavorRx when the FDA announced the relaxed oversight. That was in April, and the company within a week made EndeavorRx available on the market. "There were a lot of sleepless nights over that week," says Martucci. At least two other companies have since taken advantage of the loosening of the rules. Pear released to the public, in a limited way, its schizophrenia product candidate Pear-004, which has not yet been cleared by the FDA. Orexo, headquartered in Uppsala, Sweden, in July announced that it would launch in the United States two therapies - one for depression and one for problematic alcohol use - and accelerate the testing of its software for opioid-use disorder. The therapies are based on cognitive behavioral therapy - a kind of digitized counseling.

The relaxation of the rules gives companies an opportunity to gather data on the efficacy of their software in the real world. "It is a unique opportunity to do that and make the product available as you go," says Dennis Urbaniak, executive vice president of digital therapeutics at Orexo. His company still plans to seek FDA approval for the products it is releasing to the public, he says.

It's unclear how or whether the agency will roll back the policy once the pandemic is over. There is no requirement for companies to inform the FDA that they are taking advantage of the relaxed rules. The FDA did include a set of recommended standards that companies should meet when releasing their digital therapies to the public. "Anyone who goes forward under those pathways needs to be prepared, whether they formally notify the FDA or not, to expect that at some point the FDA will look to see what they're doing and if they are compliant with those standards," says Urbaniak.

The agency in September continued to lean into the digital therapeutics space when it announced that it was launching a Digital Health Center of Excellence. The FDA's attention on digital therapeutics during the pandemic suggests that the agency is recognizing them as legitimate, and even necessary, for human health.

\section{Emily Waltz}

Nashville, TN, USA

Published online: 3 November 2020 https://doi.org/10.1038/s41587-020-0726-6 